\title{
Synthesis of 3-[(Z)-5-Amino-1, 3, 3-trimethyl cyclohexyl methylimino]-1, 3-dihydroindol-2-one as a novel Schiff base
}

\author{
Ramakrishna Reddy.K and Mahendra.K.N* \\ Department of Chemistry, Central College Campus, Bangalore University, Bangalore-560001 \\ E-mail: mahendra_kadidal@yahoo.co.in, mahendra_kadidal@rediffmail.com \\ *Author to whom correspondence should be addressed
}

Received: 21 July 2006 / Accepted: 20 October 2006 / Published: 1 December 2006

Keywords: Isatin, Schiff base, Isophoronediamine (5-amino-1,3,3-trimethyl-cyclohexanemethylamine).

Isatin (1H-indole-2,3-dione) was first obtained by Erdman and Laurent in 1841 as a product from the oxidation of indigo by nitric and chromic acids [1]. The synthetic versatility of isatin has led to the extensive use of this compound in organic synthesis. In nature, isatin is found in plants of the genus Isatis [2], in Calanthe discolor LINDL [3]. Isatin is the biologically active chemical produced by an Altermones sp. Strain inhabiting the surface of embryos of the cardiean shrimp Palaemon macrodectylus, which protect them from the pathogenic fungus Lagenidium callinectes [4]. Schiff bases of isatin were reported to possess interesting biological activities such as anti-HIV [5-7], anticonvulsant [8], antibacterial [9-11], antiprotozoal [12,13], antifungal [14-16], antiviral [17-19], anthelminthic [20,21], antidepressant [22] anti-inflammatory and antitumour [23] activities. In view of the potent biological activities, we decided to synthesize a new isatin Schiff base by the condensation of isatin with isophoronediamine (IPDA). The Schiff bases of isatin have also been used as a ligand for complexation with various metal ions [24].The biological activities, epoxy curing studies of the new Schiff base and its chelating behaviour with various metal ions is under progress.

Ethanolic solutions of isatin (1) $(0.01 \mathrm{~mol}, 1.479)$ in $50 \mathrm{ml}$ and isophoronediamine (2) (5-amino-1,3,3trimethyl-cyclohexanemethylamine)(0.01mol, $1.8 \mathrm{ml}, 1.79 \mathrm{~g})$ in $50 \mathrm{ml}$ were mixed and refluxed for about 2 hours. The reaction mixture was evaporated to a small volume and left to cool. The resulting Schiff base (3) ligand precipitated on cooling and then was filtered off, washed with ethanol and recrystallised from ethanol. The purity of the Schiff base ligand was monitored on TLC using eluants 1:1 ethyl acetate and petroleum ether and separated by column chromatography (Yield $=90 \%$ ). 
<smiles>CC1(C)CC(N)CC(C)(CN)C1</smiles><smiles>CC1(C)CC(N)CC(C)(CN=C2C(=O)Nc3ccccc32)C1</smiles>

3

Melting point: $117^{\circ} \mathrm{C}$.

Elemental analysis: Calculated for $\mathrm{C}_{18} \mathrm{H}_{25} \mathrm{~N}_{3} \mathrm{O}$ : C, 72.14; H, 8.35; N, 14.03. Found: C, 71.95; H, 8.33; N, 13.98.

IR $\left(\mathrm{KBr}, \mathrm{cm}^{-1}\right): 1618.6(\mathrm{C}=\mathrm{N}), 1714.6(\mathrm{C}=\mathrm{O}), 3239.9(\mathrm{~N}-\mathrm{H})$.

MS (ESI, m/z): $299\left[\mathrm{M}^{+}\right]$

${ }^{1} \mathrm{H}-\mathrm{NMR}\left(400 \mathrm{MHz}, \mathrm{DMSO}-\mathrm{d}_{6}\right): 10.5$ (1H, s) 7.5-6.8 (5H, m), 2.4(2H, m), 1.5-0.65(18H, m).

${ }^{13}$ C-NMR (400MHz, DMSO-d6):23.5; 28.1; 35.3; 43.5; 45.5; 47.1; 49.2; 50.2; 57.2; 111.0; 119.9; 120.9; $122.3 ; 131.0 ; 132.0 ; 142.3 ; 168.8$.

\section{References}

1. Dasilva, J. F. M.; Garden, S. J.; Pinto, A. C. J. Braz. Chem. Soc. 2001, 12(3), 273.

2. Guo, Y.; Chen, F.; Zhongcaoyao 1986, 17, 8. (CA 104:213068f).

3. Yoshikawa, M.; Murakami, T.; Kishi, A.; Sakurama, T.; Matsuda, H.; Nomura, M.; Matsuda, H.; Kubo, M. Chem. Pharm. Bull. 1998, 46, 886.

4. Gil-Turners, M. S.; Hay, M. E.; Fenical, W. Science, 1989, 116.

5. Pandeya, S. N.; Yogeeswari, P.; Sriram, D.; De Clercq, E.; Pannecouque, C.; Witvrouw, M.

Chemotherapy, 1999, 45, 192-196.

6. Pandeya, S. N.; Sriram, D.; Nath, G.; De Clercq, E. Eur. J. Med. Chem. 2000, 35, 249-255.

7. Pandeya, S. N, Sriram, D.; Nath, G.; De Clercq, E. Arzneimittel Forschun./ Drug Res.2000,50, 55-59.

8. Sridhar, S. K.; Pandeya, S. N.; Stables, J. P.; Armes, S. K. Eur. J. Pharm. Sci. 2002, 16, 129.

9. Pandeya, S. N.; Sriram, D. Acta Pharm. Turc. 1998, 40, 33.

10. Sarangapani, M.; Reddy, V. M. Indian J. Pharm. Sci. 1994, 56, 174.

11. Varma, R. S.; Nobles, W. L. J. Pharm. Sci. 1975, 64, 881.

12. Imam, S. A.; Varma, R. S. Experientia, 1975, 31, 1287.

13. Varma, R. S.; Khan, I. A. Polish J. Pharmacol. Pharm.1977, 29, 549. 
14. Pandeya, S. N.; Sriram, D.; Nath, G.; De Clercq, E. Indian J. Pharm. Sci.1999,61, 358.

15. Pandeya, S. N.; Sriram, D.; Nath, G.; De Clercq, E. Sci.Pharm. 1999, 67, 10.

16. Pandeya, S. N.; Sriram, D.; Nath, G.; De Clercq, E. Pharm. Acta Helv. 1999, 74, 11.

17. Varma, R. S.; Nobles, W. L. J. Med. Chem. 1967, 10, 972.

18. Singh, S. P.; Shukla, S. K.; Awasthi, L. P. Curr. Sci. 1983, 52, 766.

19. Logan, J. C.; Fox, M. P.; Morgan, J. M.; Makohon, A. M.; Pfau, C. J. J. Gen. Virol. 1975, $28,271$.

20. Sarciron, S. E.; Audin, P.; Delebre, I.; Gabrion, C.; Petavy, A. F.; Paris, J. J. Pharm. Sci. 1993, 82, 605.

21. Et-savi, E. A.; Mostafa, T. B.; Mostafa, B. B. J. Egypt. Soc. Parasitol. 1998, 28, 481.

22. Seshaiah.K.S and Atmakuru.R, Biol.Pharm.Bull.2001, 24, 1149.

23. Mohanan.K and Murukan.B, Syn. and. rea. in Inorg. Met-org. and Nano-Met. Chem. 2005, 35, 837.

24. Rama Krishna Reddy.K.;Madhusudan Reddy.K and Mahendra.K.N, Ind.J.of Chem,2006,45A,377.

(c) 2006 MDPI. All rights reserved. 